\title{
Prevalence of Trichomonas vaginalis in Women Visiting 2 Obstetrics and Gynecology Clinics in Daegu, South Korea
}

\begin{abstract}
Youn-Kyoung Goo', Won-Sik Shin², Hye-Won Yang', So-Young Joo', Su-Min Song', Jae-Sook Ryu³, Won-Myung Lee ${ }^{4}$, Hyun-Hee Kong ${ }^{5}$, Won-Ki Lee ${ }^{6}$, Sang-Eun Lee ${ }^{7}$, Won-Ja Lee ${ }^{7}$, Dong-II Chung', Yeonchul Hong,**

'Department of Parasitology and Tropical Medicine, Kyungpook National University School of Medicine, Daegu 41944, Korea; ${ }^{2}$ Department of Obstetrics and Gynecology, Shinsegae Women's Hospital, Daegu 41535, Korea; ' 3 Department of Environmental Biology \& Medical Parasitology, Hanyang University College of Medicine, Seoul 04763, Korea; ${ }^{4}$ Department of Obstetrics and Gynecology, Rosemary Hospital, Daegu 41422, Korea; ${ }^{5}$ Department of Parasitology, Dong-A University College of Medicine, Busan 49201, Korea; ${ }^{6}$ Center of Biostatistics, Kyungpook National University School of Medicine, Daegu 41944, Korea; ${ }^{7}$ Division of Malaria and Parasitic Diseases, Korea National Institute of Health, Korea Centers for Diseases Control and Prevention, Osong 28159, Korea
\end{abstract}

\begin{abstract}
This study explored epidemiological trends in trichomoniasis in Daegu, South Korea. Wet mount microscopy, PCR, and multiplex PCR were used to test for Trichomonas vaginalis in vaginal swab samples obtained from 621 women visiting 2 clinics in Daegu. Of the 621 women tested, microscopy detected T. vaginalis in $4(0.6 \%)$ patients, PCR detected T. vaginalis in $19(3.0 \%)$ patients, and multiplex PCR detected T. vaginalis in $12(1.9 \%)$ patients. Testing via PCR demonstrated high sensitivity and high negative predictive value for $T$. vaginalis. Among the 19 women who tested positive for $T$. vaginalis according to PCR, $94.7 \%$ (18/19) reported vaginal signs and symptoms. Notably, more than $50 \%$ of $T$. vaginalis infections occurred in females younger than 30 years old, and $58 \%$ were unmarried. Multiplex PCR, which simultaneously detects pathogens from various sexually transmitted infections, revealed that $91.7 \%(11 / 12)$ of patients were infected with 2 or more pathogens. Mycoplasma hominis was the most prevalent co-infection pathogen with T. vaginalis, followed by Ureaplasma urealyticum and Chlamydia trachomatis. Our results indicate that PCR and multiplex PCR are the most sensitive tools for $T$. vaginalis diagnosis, rather than microscopy which has been routinely used to detect $T$. vaginalis infections in South Korea. Therefore, clinicians should take note of the high prevalence of $T$. vaginalis infections among adolescent and young women in order to prevent persistent infection and transmission of this disease.
\end{abstract}

Key words: Trichomonas vaginalis, trichomoniasis, PCR, multiplex PCR, multiple infection, sexually transmitted infection

Trichomoniasis, which is caused by the protist Trichomonas vaginalis, is one of the most common non-viral sexually transmitted infections, with an estimated prevalence of about 276 million new cases annually worldwide $[1,2]$. The prevalence of T. vaginalis varies and depends on the population, time, and region studied, but the rate is generally higher among women and older groups when compared to men or younger groups, which is in marked contrast to other sexually transmitted infections, such as Chlamydia trachomatis or Neisseria gonorrhoeae [3]. T. vaginalis infection causes vaginitis and cervicitis, and it is associated with serious complications, such as preterm birth, pelvic inflammatory disease, infertility, and an increased risk

\footnotetext{
- Received 21 October 2015, revised 4 December 2015, accepted 26 December 2015. *Corresponding author (ychong@knu.ac.kr) (c) 2016, Korean Society for Parasitology and Tropical Medicine This is an Open Access article distributed under the terms of the Creative Commons Attribution Non-Commercial License (http://creativecommons.org/licenses/by-nc/3.0) which permits unrestricted non-commercial use, distribution, and reproduction in any medium, provided the original work is properly cited.
}

of co-infection with human immunodeficiency virus [4-10]. However, more than $50 \%$ of T. vaginalis infections in women are asymptomatic and remain undiagnosed [11]. Thus, early diagnosis and treatment of T. vaginalis infections, especially asymptomatic infections, are imperative for women as this is an important public health concern.

It is also helpful to monitor changes in T. vaginalis infection rates in order to develop appropriate prevention measures. The most common clinical diagnostic test for T. vaginalis infections is microscopic evaluation of vaginal wet preparations; however, the sensitivity of wet mounts for detecting T. vaginalis is only $50-70 \%$ [12]. Cultures are the gold standard for diagnosing T. vaginalis, as cultures demonstrate increased sensitivity. However, they require at least a week for incubation, which results in a substantial delay between the patient's visit and final diagnosis $[13,14]$. Recently, nucleic acid amplification tests have been developed that use vaginal swabs, including PCR and transcription-mediated amplification. These have shown 
an improved sensitivity and specificity for the detection of $T$. vaginalis compared to microscopic examinations and culture, with sensitivity ranging from $64 \%$ to $100 \%[15,16]$. Nucleic acid amplification tests also provide an improvement in medical screening for sexually transmitted infections. Recently, a new type of PCR, multiplex PCR, has been developed, which detects several different pathogens that may cause sexually transmitted diseases [17]. However, expensive equipment and technical training make nucleic acid amplification tests impractical for routine diagnosis in the clinical setting.

In this study, we examined 621 vaginal swabs from women attending obstetrics and gynecology clinics in Daegu, South Korea using wet mount microscopy, PCR, and multiplex PCR to determine the prevalence of T. vaginalis and associated sexually transmitted infections in T. vaginalis-positive women.

From May 2013 to November 2013, 621 vaginal swab samples were obtained from women visiting 2 obstetrics and gynecology clinics in Daegu, South Korea. These were screened for trichomoniasis by wet mount microscopy and PCR and were tested for bacterial sexually transmitted diseases by multiplex PCR. The study was approved by the Institutional Review Board of Kyungpook National University Hospital (IRB no. KNUH 2013-04-051). Each participant was asked to complete a written consent form to verify informed consent and to obtain permission to collect the specimen. Data about vaginal signs and symptoms, age, and current pregnancy status were obtained through self-report. Study questionnaires and samples were labeled with a unique surveillance number delinked from patient identifying information.

Wet mount microscopy was performed at the clinical sites within 10 min of sample collection. Observation of motile trichomonads was required to classify a wet mount as positive for T. vaginalis. The study questionnaires and samples were transported to our lab using $\mathrm{eNaT}^{\mathrm{TM}}$ nucleic acid transport collection tubes (Copan Flock Technologies, Brescia, Italy). The diagnosis of T. vaginalis infection was made using PCR that targeted Tvk [17] and the $\beta$-tubulin gene [18]. The frozen swab samples were thawed, suspended in PBS, and collected by centrifugation at 13,000 $\mathrm{g}$ for $10 \mathrm{~min}$.

DNA from the cell pellets were extracted using a QIAamp DNA Mini Kit (Qiagen, Hilden, Germany) according to the manufacturer's instructions. The PCR conditions were as follows: the initiation step was conducted at $95^{\circ} \mathrm{C}$ for $5 \mathrm{~min}$, followed by 40 cycles at $90^{\circ} \mathrm{C}$ for $1 \mathrm{~min}$, then $60^{\circ} \mathrm{C}$ for $30 \mathrm{sec}$, and $72^{\circ} \mathrm{C}$ for $2 \mathrm{~min}$. A final extension step at $72^{\circ} \mathrm{C}$ was conducted for $10 \mathrm{~min}$. The PCR primer set Tvk 3/7 (Tvk 3, 5'-ATTGTCGAACATTGGTCTTACCCTC-3'; Tvk 7, 5'-TCTGTGCCGTCTTCAAGTATGC-3') [17] amplified the 261 base pair (bp) products. The PCR primer set $\beta$-tubulin 9/2 ( $\beta$-tubulin 9, 5'-CATTGATAACGAAGCTCTTTACGA-3'; $\beta$-tubulin 2, 5'-GCATGTTGTGCCGGACATAACCAT-3') [18] amplified DNA products of $112 \mathrm{bp}$ from T. vaginalis genomic DNA. PCR using the Tvk $3 / 7$ primer set and multiplex PCR were performed in duplicate. If the results of the duplicate reactions of each test were not concordant, a third amplification and detection was performed to resolve the discrepancy. In cases of discrepancies between PCR and multiplex PCR results, PCR using the $\beta$-tubulin 9/2 primer set was performed, and these PCR results (both Tvk $3 / 7$ and $\beta$-tubulin 9/2 PCR-positive) were considered final (positive for T. vaginalis).

The test sensitivities and specificities were determined using PCR as the gold standard. The percentage sensitivities and specificities with 95\% confidence intervals were calculated with MedCalc version 7.0 (MedCalc Software bvba, Ostend, Belgium). Differences in the sensitivities and specificities were compared with McNemar's normal approximation test using SAS 9.3 (SAS Institute Inc., Cary, North Carolina, USA). The degree of agreement was determined using Kappa statistics in SAS 9.3 (SAS Institute Inc.). Results were considered statistically significance for $P$-values less than 0.001 . In this study, the lower limit of T. vaginalis detection by PCR using Tvk $3 / 7$ and $\beta$-tubulin $9 / 2$ was found to be 1 organism per reaction, which was consistent with a previous report [18]. The presence of multiple infections with T. vaginalis, including $C$. trachomatis, $N$. gonorrhoeae, Mycoplasma genitalium, Ureaplasma urealyticum, and Mycoplasma hominis [19], were simultaneously detected using multiplex PCR (STD6 ACE Detection Kit, Seegene, Seoul, Korea).

A total of 621 subjects who visited 2 obstetrics and gynecology clinics in Daegu, South Korea were included in this study. The mean age of all patients was 36.2 years old (range 16-64 years), although the group was age-diverse (Table 1 ). The subjects included 199 (32\%) single women and 422 (68\%) married women; of these, 41 women were pregnant (6.6\%) (Table 1). Among all 621 subjects, 557 women reported experiencing vaginal signs and symptoms, including vaginal discharge (473 women, 76.2\%), itching (283 women, 45.6\%), odor (199 women, $32.0 \%$ ), dysuria (21 women, 3.38\%), and abdominal pain (21 women, 3.4\%) (Table 1).

Microscopic examination of wet mounts diagnosed T. vagi- 
nalis in $4(0.6 \%)$ women. PCR detected T. vaginalis in 19 (3.1\%) women. T. vaginalis was detected via multiplex PCR in 12 (1.9\%) women (Table 2). The PCR results (19 positives and 602 negatives) were used as the gold standard for detecting T. vaginalis, and as such, the PCR results were used as a baseline to assess the accuracy of the other diagnostic tests. Accordingly, the sensitivity, specificity, positive predictive value, and negative predictive value were calculated for each test.

Microscopic examinations were positive for 4 specimens, resulting in a sensitivity of $15.8 \%$ (95\% CI, 4.2-40.5\%) and a specificity of $99.8 \%$ (95\% CI, 98.9-100\%) (Table 2). All 4 positive specimens detected by microscopy were confirmed via PCR and multiplex PCR. The results obtained by multiplex PCR were positive for 12 specimens, resulting in a sensitivity of $57.9 \%$ (95\% CI, $79.1-100 \%)$ and a specificity of $99.8 \%$ (95\% CI, 49.2-75.3\%) (Table 2). All 12 T. vaginalis-positive

Table 1. Analysis of Trichomonas vaginalis infection status according to sociodemographic characteristics and urogenital symptoms $(\mathrm{N}=621)$

\begin{tabular}{llrr}
\hline Factor & Characteristic & $\begin{array}{c}\text { No. of } \\
\text { patients }\end{array}$ & $\begin{array}{r}\text { T. vaginalis } \\
\text { prevalence (\%) }\end{array}$ \\
\hline Overall & & 621 & $19(3.1)$ \\
Age (yr) & $<20$ & 14 & $2(14.3)^{\mathrm{a}}$ \\
& $20-29$ & 197 & $8(4.1)^{\mathrm{a}}$ \\
& $30-39$ & 171 & $3(1.8)$ \\
& $40-49$ & 157 & $4(2.5)$ \\
& $50-59$ & 63 & $2(3.2)$ \\
Marital status & $60-69$ & 19 & $0(0.0)$ \\
& Single & 188 & $10(5.3)^{\mathrm{a}}$ \\
& Married & 422 & $8(1.9)$ \\
Pregnancy status & Yes & 1 & $0(0.0)$ \\
& Divorced & 9 & $1(11.1)$ \\
& No & 11 & $2(18.2)$ \\
Urogenital & Missing & 74 & $14(18.9)$ \\
symptoms & None & 16 & $3(18.8)$ \\
& Vaginal discharge & 64 & $1(1.6)$ \\
& Vaginal itching & 283 & $14(3.0)$ \\
& Vaginal odor & 199 & $10(3.2)$ \\
& Dysuria & 21 & $2(5.0)$ \\
& Lower abdominal pain & 21 & $0(0.0)$ \\
\hline
\end{tabular}

ap $<0.05$. specimens detected by multiplex PCR were confirmed using PCR. In addition, 7 of the 19 samples found to be positive for T. vaginalis using PCR $(7 / 19,36.4 \%)$ were negative according to multiplex PCR and thus considered to be false negatives. Microscopy and multiplex PCR both showed high specificity (99.8\%, 95\% CI: 98.9-100\%). However, sensitivity was distinct between the 2 methods. Compared to microscopic examination, multiplex PCR demonstrated a significantly higher sensitivity $(P<0.001$ vs microscopy) and better agreement (concordance, 94.9\%; kappa value, 0.865 ) with PCR than microscopy (Table 2).

These findings were consistent with previous reports in which the newly developed nucleic acid amplification tests, such as PCR and multiplex PCR, were able to accurately detect more samples infected with $T$. vaginalis compared to traditional techniques, including microscopic examination of wet mounts and cultures [18-20]. This result is noteworthy since misdiagnosis of $T$. vaginalis as a result of using a less accurate diagnostic method would result in a persistent infectious state, and may subsequently lead to serious complications as well as possibly broad transmission of the infection. In addition, administration of metronidazole is an effective treatment for $T$. vaginalis infection; therefore, diagnosis by a highly sensitive method followed by rapid treatment would be a good strategy to control this disease.

In this cohort of female patients who visited obstetrics and gynecology clinics, the prevalence of T. vaginalis infection was 3.1\% (19/621) according to PCR testing. To date, several studies regarding T. vaginalis infection in South Korea have reported varying prevalences of $T$. vaginalis according to the specific investigators and study populations, as reviewed by Ryu and Min [21]. Similarly, the prevalence of T. vaginalis infection showed variability in 2 recent studies, with a rate of $2.6 \%$ in Cheonan and $0.1 \%$ in Seoul, South Korea $[17,22]$. Our results showed an infection rate of 3.0\% (19/621), which is similar to the rate found by the surveillance study performed in Cheonan. This is likely due to the fact that our research in Daegu and

Table 2. Comparison of results according to diagnostic method among 621 specimens

\begin{tabular}{|c|c|c|c|c|c|c|c|}
\hline Assay & No. positive & No. negative & Sensitivity (95\% Cl) & Specificity (95\% Cl) & PPV $(95 \%$ Cl) & NPV (95\% Cl) & $\begin{array}{c}\text { \% agreement with } \\
\text { nested PCR (Kappa) }\end{array}$ \\
\hline Microscopy & 4 & 617 & $15.8(4.2-40.5)$ & 99.8 (98.9-100) & $75.0(21.9-98.7)$ & $97.4(95.7-98.5)$ & $81.9(0.624)$ \\
\hline Multiplex PCR & 12 & 609 & $57.9(79.1-100)$ & 99.8 (98.9-100) & 91.7 (59.8-99.6) & $98.7(97.3-99.4)$ & $94.9(0.865)$ \\
\hline
\end{tabular}

PPV, positive predictive value; NPV, negative predictive value; $\mathrm{Cl}$, confidence interval. 
the study conducted in Cheonan both assessed samples from symptomatic patients who visited the hospital, while the study in Seoul assessed samples from healthy women who visited the hospital for a routine health checkup.

The median age of the 19 females with T. vaginalis-positive specimens was 31.9 years (range 16-53 years). As shown in Table 1, peak T. vaginalis infection incidence was found in the younger study group ( $<30$ years). Notably, $10(52.6 \%)$ of the 19 T. vaginalis-infected subjects were under 30 years old, and the positive rate in the age group $<30$ years $(4.8 \%, 10 / 211)$ was higher than the rate in the age group over age $30(2.2 \%, 9 / 410)$ $(P<0.05)$. A similar tendency was reported in past studies, as reviewed by Ryu and Min [21], in that patients between 20-34 years old were most affected by T. vaginalis among patients of all ages who visited obstetrics and gynecology practices. However, these results are inconsistent with recent studies conducted in other countries in which the incidence of $T$. vaginalis increased by age $[23,24]$. The high positive rate in the younger study group ( $<20$ years) might be a result of the social atmosphere in South Korea in which young women often feel uncomfortable about going to obstetrics and gynecology clinics and thereby neglect the disease until vaginal symptoms and signs become serious. Since T. vaginalis infections may become persistent without treatment, this tendency to avoid care should be considered, as early diagnosis and treatment at a young age are necessary to prevent the transmission of this disease.

An examination of marital status showed that 5.3\% (10/188) of T. vaginalis-positive patients were single, and 1.9\% (8/422) were currently or previously married, demonstrating that $T$. vaginalis is more prevalent in single women $(P<0.05)$. T. vaginalis infection in this cohort was more prevalent among adolescent and younger unmarried women. According to an analysis of the self-administered questionnaires, only 1 patient reported no symptoms among the 19 patients who were positive for $T$. vaginalis. On the other hand, $94.7 \%$ (18/19) of T. vaginalis-infected female patients reported urogenital symptoms, such as vaginal discharge $(73.7 \%, 14 / 19)$, vaginal itching $(47.4 \%$, 9/19), odor (52.6\%, 10/19), and dysuria (10.5\%, 2/19).

According to multiplex PCR tests for simultaneous detection of pathogens that cause sexually transmitted infections, T. vaginalis was detected in 12 out of 621 samples (1.9\%) (Table 3). As shown in Table 2, multiplex PCR was less sensitive for $T$. vaginalis detection than PCR (which detected 19 positive samples). While previous reports have suggested possible limitations of multiplex PCR, which include PCR drift by stochastic
Table 3. Frequency of comorbid sexually transmitted infections in Trichomonas vaginalis-positive samples according to multiplex PCR

\begin{tabular}{lcc}
\hline Pathogens & $\begin{array}{c}\text { No. } \\
\text { patients }\end{array}$ & $\begin{array}{c}\text { Percentage } \\
(\%)\end{array}$ \\
\hline Single infection with $T$. vaginalis & 1 & 8.3 \\
Double infection & & \\
$\quad$ T. vaginalis- $U$. urealyticum & 2 & 16.7 \\
T. vaginalis- $M$. hominis & 1 & 8.3 \\
$T$. vaginalis- $N$. gonorrhoeae & 1 & 8.3 \\
Triple infection & & \\
$T$ T. vaginalis- $M$. hominis- $U$. urealyticum & 4 & 33.4 \\
T. vaginalis-M. hominis- - C. trachomatis & 3 & 25.0 \\
Total & 12 & 100.0 \\
\hline
\end{tabular}

fluctuation due to the interaction of PCR reagents or by competitive inhibition from PCR selection [25], multiplex PCR is useful when detecting comorbid pathogens simultaneously, demonstrating both high sensitivity and specificity [26].

T. vaginalis is frequently co-localized with bacterial vaginosis, which is an established risk factor for adverse reproductive outcomes, such as pregnancy loss, premature rupture of membranes, and preterm labor $[4,27]$. Among the 12 T. vaginalispositive samples detected by multiplex PCR, 91.7\% (11/12) were positive for co-infection. Moreover, 7 (58.3\%) of the samples were positive for triple infections, which is defined as being infected with T. vaginalis and 2 different pathogens among the 5 pathogens (C. trachomatis, N. gonorrhoeae, M. genitalium, U. urealyticum, and M. hominis). Four (33.3\%) samples were positive for double infections, defined as being infected with $T$. vaginalis and 1 pathogen among those 5 pathogens. As shown in Table 3, M. hominis (66.7\%, 8/12 samples) was the most prevalent co-infection pathogen, followed by $U$. urealyticum $(50 \%, 6 / 12)$, and $C$. trachomatis $(25 \%, 3 / 12)$. T. vaginalis infection in conjunction with both $M$. hominis and $U$. urealyticum was the most common triple infections (33.3\%, $4 / 12$ ). Since these pathogens have a common route of transmission, i.e., via the vagina, concurrent sexually transmitted infections, including M. hominis, U. urealyticum, C. trachomatis, and $N$. gonorrhoeae, are frequently detected in persons with $T$. vaginalis infection $[28,29]$.

Comorbid infection with these pathogens may be more prevalent among female T. vaginalis-positive patients due to their symbiotic association with $T$. vaginalis, as previously suggested. In the present study, co-infections with T. vaginalis (both double as well as triple infections) were observed in 11 out of 12 T. vaginalis-positive samples diagnosed by multiplex 
PCR. Consistent with the high prevalence of T. vaginalis infection in young women, $6(50 \%)$ of the 12 T. vaginalis-positive patients that were detected by multiplex PCR assay were aged $<30$ years, which shows that there was a high percentage of co-infection with other sexually transmitted infections. Moreover, our previous surveillance studies of male patients attending a primary care urology clinic in the same region (Daegu) and time period found a T. vaginalis prevalence of $4 \%$ (8/201), which was slightly higher than the rate among female patients [30]. However, T. vaginalis was significantly more prevalent among older men ( $\geq 40$ years, $87.5 \%$ ) than among relatively younger men $(<40$ years, $11.5 \%)$, which is opposite of the distribution pattern of T. vaginalis prevalence in women by age. A limitation of the present study is the relatively small sample size, therefore providing only limited surveillance results. Nevertheless, these results provide good evidence for a bipolarized distribution of the prevalence of T. vaginalis infection according to age between male and female patients.

In this study, the prevalence of T. vaginalis infection in Dae$\mathrm{gu}$, South Korea was evaluated after determining the efficacy of 3 diagnostic methods for detecting $T$. vaginalis. Infection with $T$. vaginalis was detected in $3.1 \%$ of the study population in Daegu via PCR. Interestingly, a higher infection rate was observed in the younger study group ( $<30$ years), and a high percentage of the total study population was co-infected with other sexually transmitted infections. Further studies are required to monitor the prevalence of T. vaginalis in adolescents and young women to prevent the transmission and burden from this disease.

\section{ACKNOWLEDGMENTS}

This study was supported by a grant from the Korea National Institute of Health (no. 2013-E54010-00).

\section{CONFLICT OF INTEREST}

We have no conflict of interest related to this work.

\section{REFERENCES}

1. Gerbase AC, Rowley JT, Heymann DH, Berkley SF, Piot P. Global prevalence and incidence estimates of selected curable STDs. Sex Transm Infect 1998; 74(suppl 1): S12-S16.

2. World Health Organization (WHO). Global incidence and prevalence of selected curable sexually transmitted infec- tions-2008. Geneva, Switzerland. WHO. 2012.

3. Ginocchio C, Chapin K, Smith J, Aslanzadeh J, Snook J, Hill C, Gaydos C. Prevalence of Trichomonas vaginalis and coinfection with Chlamydia trachomatis and Neisseria gonorrhoeae in the United States as determined by the Aptima Trichomonas vaginalis nucleic acid amplification assay. J Clin Microbiol 2012; 50: 26012608.

4. Fichorova RN. Impact of T. vaginalis infection on innate immune responses and reproductive outcome. J Reprod Immunol 2009; 83: 185-189.

5. Cotch M, Pastorek Jn, Nugent R, Hillier S, Gibbs R, Martin D, Eschenbach D, Edelman R, Carey J, Regan J, Krohn M, Klebanoff M, Rao A, Rhoads G. Trichomonas vaginalis associated with low birth weight and preterm delivery. The Vaginal Infections and Prematurity Study Group. Sex Transm Dis 1997; 24: 353-360.

6. Kigozi GG, Brahmbhatt H, Wabwire-Mangen F, Wawer MJ, Serwadda D, Sewankambo N, Gray RH. Treatment of Trichomonas in pregnancy and adverse outcomes of pregnancy: a subanalysis of a randomized trial in Rakai, Uganda. Am J Obstet Gynecol 2003; 189: 1398-1400.

7. Moodley P, Wilkinson D, Connolly C, Moodley J, Sturm AW. Trichomonas vaginalis is associated with pelvic inflammatory disease in women infected with human immunodeficiency virus. Clin Infect Dis 2002; 34: 519-522.

8. Rendón-Maldonado J, Espinosa-Cantellano M, Soler C, Torres J, Martínez-Palomo A. Trichomonas vaginalis: in vitro attachment and internalization of HIV-1 and HIV-1-infected lymphocytes. J Eukaryot Microbiol 2003; 50: 43-48.

9. Guenthner PC, Secor WE, Dezzutti CS. Trichomonas vaginalis-induced epithelial monolayer disruption and human immunodeficiency virus type 1 (HIV-1) replication: implications for the sexual transmission of HIV-1. Infect Immun 2005; 73: 41554160 .

10. Kissinger P, Amedee A, Clark RA, Dumestre J, Theall KP, Myers L, Hagensee ME, Farley TA, Martin DH. Trichomonas vaginalis treatment reduces vaginal HIV-1 shedding. Sex Transm Dis 2009; 36: 11-16.

11. Workowski KA, Berman S. Sexually transmitted diseases treatment guidelines, 2010. MMWR Recomm Rep 2010; 59: 1-110.

12. Radonjic IV, Dzamic AM, Mitrovic SM, Arsenijevic VSA, Popadic DM, Zec IFK. Diagnosis of Trichomonas vaginalis infection: the sensitivities and specificities of microscopy, culture and PCR assay. Eur J Obstet Gynecol Reprod Biol 2006; 126: 116-120.

13. Caliendo A, Jordan J, Green A, Ingersoll J, Diclemente R, Wingood G. Real-time PCR improves detection of Trichomonas vaginalis infection compared with culture using self-collected vaginal swabs. Infect Dis Obstet Gynecol 2005; 13: 145-150.

14. Huppert JS, Mortensen JE, Reed JL, Kahn JA, Rich KD, Miller WC, Hobbs MM. Rapid antigen testing compares favorably with transcription-mediated amplification assay for the detection of Trichomonas vaginalis in young women. Clin Infect Dis 2007; 45: 194-198.

15. Lawing LF, Hedges SR, Schwebke JR. Detection of trichomonosis 
in vaginal and urine specimens from women by culture and PCR. J Clin Microbiol 2000; 38: 3585-3588.

16. Schirm J, Bos PA, Roozeboom-Roelfsema IK, Luijt DS, Möller LV. Trichomonas vaginalis detection using real-time TaqMan PCR. J Microbiol Methods 2007; 68: 243-247.

17. Kim Y, Kim J, Lee KA. Prevalence of sexually transmitted infections among healthy Korean women: implications of multiplex PCR pathogen detection on antibiotic therapy. J Infect Chemother 2014; 20: 74-76.

18. Van Der Pol B, Williams JA, Taylor SN, Cammarata CL, Rivers CA, Body BA, Nye M, Fuller D, Schwebke JR, Barnes M. Detection of Trichomonas vaginalis DNA by use of self-obtained vaginal swabs with the BD ProbeTec Qx assay on the BD Viper system. J Clin Microbiol 2014; 52: 885-889.

19. van der Schee C, van Belkum A, Zwijgers L, van der Brugge E, O'neill EL, Luijendijk A, van Rijsoort-Vos T, van der Meijden WI, Verbrugh H, Sluiters HJ. Improved diagnosis of Trichomonas vaginalis infection by PCR using vaginal swabs and urine specimens compared to diagnosis by wet mount microscopy, culture, and fluorescent staining. J Clin Microbiol 1999; 37: 4127-4130.

20. Wendel KA, Erbelding EJ, Gaydos CA, Rompalo AM. Trichomonas vaginalis polymerase chain reaction compared with standard diagnostic and therapeutic protocols for detection and treatment of vaginal trichomoniasis. Clin Infect Dis 2002; 35: 576-580.

21. Ryu JS, Min DY. Trichomonas vaginalis and trichomoniasis in the Republic of Korea. Korean J Parasitol 2006; 44: 101-116.

22. Kim JK. Epidemiological trends of sexually transmitted infections among women in Cheonan, South Korea, 2006-2012. J Microbiol Biotechnol 2013; 23: 1484-1490.

23. Meites E, Llata E, Braxton J, Schwebke JR, Bernstein KT, Pathela P, Asbel LE, Kerani RP, Mettenbrink CJ, Weinstock HS. Trichomonas vaginalis in selected US sexually transmitted disease clinics: testing, screening, and prevalence. Sex Transm Dis 2013; 40: 865-
869.

24. Sutton M, Sternberg M, Koumans EH, McQuillan G, Berman S, Markowitz $\mathrm{L}$. The prevalence of Trichomonas vaginalis infection among reproductive-age women in the United States, 20012004. Clin Infect Dis 2007; 45: 1319-1326.

25. Kosters K, Reischl U, Schmetz J, Riffelmann M, Wirsing von Konig CH. Real-time LightCycler PCR for detection and discrimination of Bordetella pertussis and Bordetella parapertussis. J Clin Microbiol 2002; 40: 1719-1722.

26. McIver CJ, Rismanto N, Smith C, Naing ZW, Rayner B, Lusk MJ, Konecny P, White PA, Rawlinson WD. Multiplex PCR testing detection of higher-than-expected rates of cervical Mycoplasma, Ureaplasma, and Trichomonas and viral agent infections in sexually active australian women. J Clin Microbiol 2009; 47: 13581363.

27. Brotman RM, Klebanoff MA, Nansel TR, Yu KF, Andrews WW, Zhang J, Schwebke JR. Bacterial vaginosis assessed by gram stain and diminished colonization resistance to incident gonococcal, chlamydial, and trichomonal genital infection. J Infect Dis 2010; 202: 1907-1915.

28. Samra Z, Rosenberg S, Madar-Shapiro L. Direct simultaneous detection of 6 sexually transmitted pathogens from clinical specimens by multiplex polymerase chain reaction and auto-capillary electrophoresis. Diagn Microbiol Infect Dis 2011; 70: 17-21.

29. White MJ, Sadalla JK, Springer SR, Counselman FL. Is the presence of Trichomonas vaginalis a reliable predictor of coinfection with Chlamydia trachomatis and/or Neisseria gonorrhoeae in female ED patients? Am J Emerg Med 2005; 23: 127-130.

30. Seo JH, Yang HW, Joo SY, Song SM, Lee YR, Ryu JS, Yoo ES, Lee WK, Kong HH, Lee SE, Lee WJ, Goo YK, Chung DI, Hong Y. Prevalence of Trichomonas vaginalis by PCR in men attending a primary care urology clinic in South Korea. Korean J Parasitol 2014; 52: 551-555. 